\title{
SOME SUMMER SUN
}

\section{Paolo Tiausas}

Ateneo de Manila University paolotiausas@gmail.com

\section{About the Author}

Paolo Tiausas completed his undergraduate studies at the Ateneo de Manila University with a Major in Creative Writing and a Minor in Literature-Filipino. He was selected as a fellow for poetry for the 16th Ateneo Heights Writers Workshop in 2010, the 11th Ateneo National Writers Workshop in 2012, and the 14th IYAS National Writers Workshop in 2014. His poems have appeared in Heights, Softblow, transit, and The Philippines Free Press. He is currently teaching part-time in the Fine Arts Program of Ateneo de Manila University. 


\section{SCENE}

Everything calculates our movements. Two strangers in an empty street. Except a concrete field. Nothing transpires, the air quite clear. Days inside which the heat resides. A van swerving towards \& away with the same intensity each replay. Revealing faces seconds before they melt. Sunlight's never this treacherous it's each dangerous glint in each reflector. The sidewalk heavy. That much true. The color an expanse set blue on fire, as though gas set off by foreign matter. Little match girl. Signs of warning, things falling, construction's this dangerous site for witnesses. So situate ourselves. Situate this phase between others. I am going to tell you a story of how everything... 


\section{PERIMETER}

A child tells a story, this avenue strikes off a character from her list. In that way she knows all, sees all. Synapses spread throughout the map, street corners, sewages. So today's protagonist is a spirit medium. So today's a child with a locket decades older than this story. A death album. Assortment of buildings, structures line the street, the meeting place this small semi-park. Around the characters, a staged design of lights \& sounds start to carry the plot. The child begins, "everything calculates our movements..." as a van crushes him against a convenience store. Strike one off. The third or fourth. Again. As if all is space and time is less. As if all is time and space is less. 


\section{CONTEXT}

The expanse of carved stone is endless. Split in measured groups, a dispersed crowd patrols the scene. The time now is afternoon. Quiet hum of engines, occasional impatient horn. Not everyone tries to understand death, you see. Not every one. Days before, we talked about Life's a cycle and Everyday's just a re-enactment. Days before, I thought about the same exasperated sigh heaving days later. As if set in stone, heaving an endless repetition. Now? A moment. Would your answer alter courses of vehicles, those choosing this day to sway off cement? A new set of problems. All while your body's still bent, unaware of this. Grand, rehearsed, cavalier. 


\section{DiAlogue}

I'd like to stay a while. Bite marks from jagged unseen teeth have appeared on my wrist. My feet drag in attempts to walk. Any direction's fine, but you see I'm so exhausted. As if my body's become too much a body, less me. When did I gain so much weight? When did I start feeling for my face? When did this happen? And when did this happen? I thought circumstance. I thought narrative and once that fails I will be dialogue itself. When did my name change? When did it meet? Something seethes. There is a city where everyone sleeps. Nothing more, nothing less than a scene with me in its center. Every single thing moving except me. So I stay here lately. 


\section{REVISION}

The mind's an exact knife. The material hates me. Carving details in this suffering, all your deaths well-timed breaks in a process. I tell myself focus, I am making something better. Narrative of a hero to alter whichever question $\&$ answer. As if the long tired shadows of the x-storey buildings were signalling an end. The end. Some summer sun runs away. A few children start to play. From stage left comes a van rampaging right where the victim stands. Some knives can't dissect a story enough, so conclusions arrive \& disappear of their own accord. Set another word on fire, then another. All this meaning no dire things. Only myths. The gods who were told. How every thing is better. Better not to hold. 